Irish Math. Soc. Bulletin

Number 69, Summer 2012, 57-59

ISSN 0791-5578

\title{
Seán Dineen: Analysis - A Gateway to Understanding Mathematics, World Scientific, 2012, ISBN:978-9814401388
}

\author{
REVIEWED BY TOM CARROLL
}

The book under review is a first course not only in analysis and calculus but in the culture of mathematics. It grew from lecture notes for a mathematics course aimed at Economics and Finance students at University College Dublin and caters not only for mathematics students but for students whose area of primary interest lies outside mathematics. It is clear that the author believes that all students, including those who would typically be classed by a mathematics department as taking a 'service course', should be expected to understand the principles of mathematics and be skilled in their use.

Dineen paints on a broad canvas. The topics standard to all calculus and analysis textbooks are covered completely and in detail number, function, limits and continuity, sequences and series, differentiation, integration, applications - with the approach being thorough right from the beginning. The reader is encouraged to think about each topic from different points of view. Rather than assuming the role of the omniscient author who providentially introduces material in advance of needing it, Dineen deals with issues only as they arise and introduces new mathematics only as it is needed. For example, though proofs are centre stage throughout the book, readers pick up proof techniques by degrees and in a manner commensurate with their growing mathematical maturity and confidence. That this approach works is partly due to the historical narrative which runs parallel to the mathematics and which explains how central concepts, such as number, function, limit, came into focus only gradually and were used implicitly long before their modern definitions solidified, often long before it was even realized that a precise definition might be needed. Pen pictures of the main contributors to the development of mathematics give the narrative a human feel and reinforce the message that understanding this mathematics takes

Received on 7-8-2012. 
time and commitment. Teachers' expectation of engagement on the part of students needs to be matched by a reciprocal commitment to engage with students and to bring students along with them. Dineen does this through a non-linear mathematical narrative. In the first chapter on Quadratic Functions, the rules of algebra, cancelation, square roots, functions are used freely, even if they will be formally introduced only later on. Though the exponential function and the logarithm function (modulo the intermediate value theorem which is proved later) are first formally defined in Chapter 7, these functions are introduced informally in Chapter 2 and are used freely throughout the book. Real Numbers feature right from the start that positive numbers have a square root is used in Chapter 1, the completeness axiom is the basis of Chapter 6 where all the main properties of supremum and infimum of sets of real numbers are proved - even if the completeness axiom and the construction of the real numbers comes later in Chapter 12. It is in this sense that the narrative is 'non-linear'. It has taken great care and thought on the part of the author to ensure that this approach works logically, which it most certainly does. Dineen is frank about his approach. He introduces concepts gradually, informally at first, with an emphasis on understanding rather than absolute rigour. He aims to 'blend intuitive techniques and rigourous definitions' with the rigor coming later, often motivated by the historical realization that clarity and precision would be essential if further progress was to be made (cf, for example, Berkeley's criticisms of Newton and Leibniz's calculus, which Dineen discusses in detail). Dineen's approach steers a careful course between the ubiquitous calculus tome and a potentially dry first course in mathematical analysis and, in so doing, is more reflective of the way we learn mathematics. Let me be entirely clear that, though the mathematical development often moves ahead of itself only to regroup later, the narrative is entirely consistent and leaves no loose ends.

To conclude, a technical word or two about the author's mathematical choices. The rational numbers are constructed from the positive rational numbers which are in turn constructed directly from the natural numbers, so that the integers come after the rationals in Dineen's development. This works very well and is, of course, a perfect opportunity to bring in equivalence relations. Countability is covered in the context of number and function. Analysis is based on 
sequences, which avoids any $\epsilon-\delta$ arguments. In fact, Dineen avoids $\epsilon$ entirely by restricting himself first to monotonic sequences, defining the limit of a bounded monotonic sequence to be the supremum if it is increasing, or the infimum if it is decreasing, of the set of real numbers which occur in the sequence. Having consolidated this notion through a variety of examples and results, Dineen defines a general sequence to be convergent if it lies between an increasing and a decreasing sequence which are convergent and have the same limit. All aspects of infinite series are covered in detail. From here he naturally defines continuity of a function at a point to be sequential continuity. I particularly enjoyed Chapter 10 on the construction of the real numbers using sections of the dyadic rationals and a 'bisection principle'. The last sections of the book cover first the derivative and its applications, then the Riemann integral for continuous functions and its applications. A notable feature of the book are the numerous well thought out, interesting exercises at the end of each chapter, with solutions provided at the end of the book.

Tom Carroll is a senior lecturer in mathematics at University College Cork. His main areas of mathematical interest are complex analysis and potential theory

School of Mathematical Sciences, University College, Cork

E-mail address: t.carroll@ucc.ie 